\title{
A Novel Single-Inductor Injection-Locked Frequency Divider by Three With Dual-Injection Secondary Locking
}

\author{
Alessandro Garghetti, Member, IEEE, Andrea L. Lacaita ${ }^{\circledR}$, Fellow, IEEE, \\ and Salvatore Levantino ${ }^{\circledR}$, Senior Member, IEEE
}

\begin{abstract}
The paper presents a comprehensive analysis of signal intermixing taking place across the injectors of frequencydivide-by-three circuits with divide-by-two secondary locking. The analytical results confirmed by circuit simulations provide an insightful understanding of the circuit operation, and inspire the design of a novel single-inductor injection-locked frequency divider (ILFD) by three, where injection is reinforced by a dual-injection scheme with no penalty in power dissipation. The novel circuit, when benchmarked against existing ILFD topologies, optimized in a $65-\mathrm{nm}$ LP CMOS process, shows about a three-time larger locking range with respect to the single-inductor divider by three with a floating-source injector, and about a $40 \%$ improvement with respect to the single-inductor divider by three with divide-by-two locking, for the same power dissipation. The novel topology has been adopted in a $15-\mathrm{GHz}$ divider by three for a $5 \mathrm{G}$ radio-frequency synthesizer, reaching a $23.6 \%$ locking range at $1.56-\mathrm{mW}$ dc power, featuring one of the best performances among divide-by-three ILFDs and a compact size of only $0.09 \mathrm{~mm}^{2}$.
\end{abstract}

Index Terms-CMOS, injection-locked frequency divider (ILFD), divide-by-3, locking range, low power, single inductor, radio-frequency (RF), frequency synthesis, phase-locked loop.

\section{INTRODUCTION}

I $\mathrm{N}$ RECENT years, wireless communications have experienced an incredible growth, and a clear trend exists towards higher working frequencies and millimeter-wave operating bands. In these applications, the frequency divider is a critical block and injection-locked frequency dividers (ILFDs) are attracting more interest than other kinds of architecture, thanks to their ability to obtain both low power consumption and low phase noise performance at high frequencies.

LC-based ILFD circuits are essentially LC-tank oscillators that are locked to an external reference by periodically injecting a current tone into the resonator. To this aim, the most used

Manuscript received May 6, 2018; revised August 24, 2018; accepted September 14, 2018. This paper was recommended by Associate Editor G. Di Capua. (Corresponding author: Salvatore Levantino.)

A. Garghetti was with the Dipartimento di Elettronica, Informazione e Bioingegneria, Politecnico di Milano, 20133 Milan, Italy. $\mathrm{He}$ is now with SK Hynix Inc., 20864 Agrate Brianza (MB), Italy (e-mail: alessandro1.garghetti@mail.polimi.it).

A. L. Lacaita and S. Levantino are with the Dipartimento di Elettronica, Informazione e Bioingegneria, Politecnico di Milano, 20133 Milan, Italy (e-mail: salvatore.levantino@polimi.it).

Color versions of one or more of the figures in this paper are available online at http://ieeexplore.ieee.org.

Digital Object Identifier 10.1109/TCSI.2018.2871178

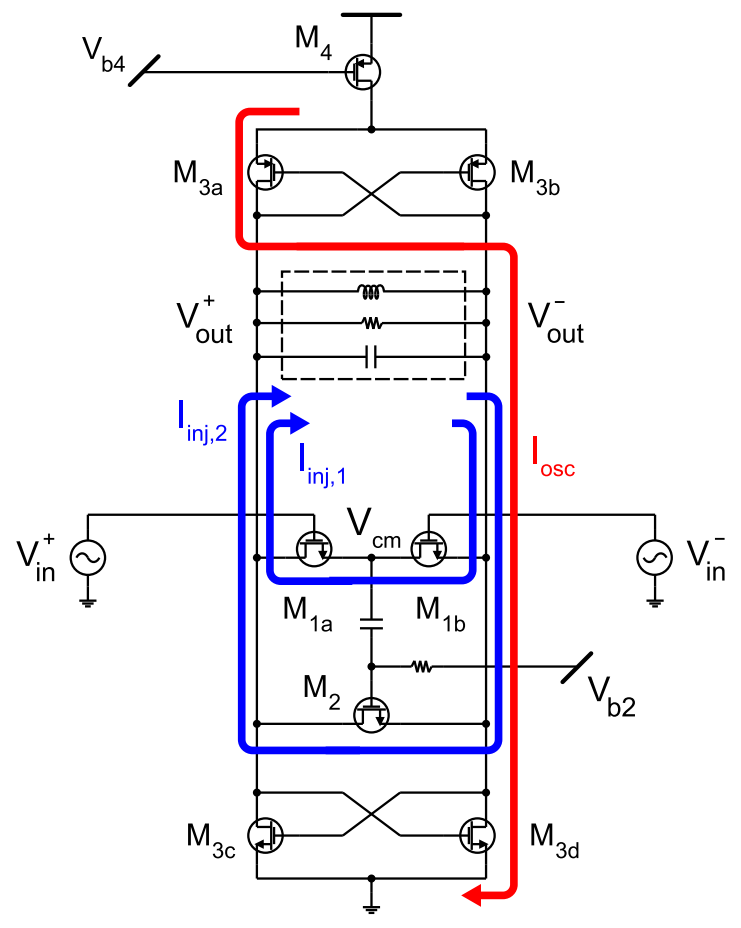

Fig. 1. ILFD by three with divide-by-two locking [2]. The arrows denote the injection currents $I_{i n j, 1}$ and $I_{i n j, 2}$, and the oscillation current $I_{o s c}$, that is in phase with the output differential voltage $\left(V_{\text {out }}^{+}-V_{\text {out }}^{-}\right)$. The positions of the source terminals of $M_{1 a}, M_{1 b}$ and $M_{2}$ refer to the case of a positive output differential voltage.

injection mechanism is the direct one, in which the injector is directly placed in parallel to the tank. To obtain a division factor of three, the injector is usually implemented with a floating-source differential structure [1].

Most of the improved ILFD topologies recently reported in literature increase performance by using additional inductors [3]-[5], however at the cost of larger area occupation, which is a limited resource in low-cost CMOS millimeter-wave radios. An alternative approach is to keep a single-inductor topology while increasing the injection current amplitude by suitable topological improvements. This is the case of the divide-by-three ILFD with divide-by-two locking, proposed by Wu et al. [2] and illustrated in Fig. 1. In this circuit, the floating-source injector $\left(M_{1 a}, M_{1 b}\right)$ generates a first 
injection current, $I_{i n j, 1}$. A second injector, $M_{2}$, is then added in parallel, driven by a down-converted voltage signal at the source terminal of the floating-source injector, thus generating a further injection current, $I_{i n j, 2}$, which adds in phase to the first one.

This paper presents a novel comprehensive analysis of the circuit in Fig. 1, describing the intermixing signal generated across both the floating-source and the divide-by-two injectors (Sect. II), first relying on a small-signal-injection approximation and then extending the analysis to large-signal injection. The results, confirmed by circuit simulations, inspire a new divider topology (Sect. III), where a pMOS tail transistor $M_{4}$ is used as an extra divide-by-two injection path [6]. A thorough comparison between the performance of conventional and novel divide-by-three ILFD topologies in 65-nm CMOS is provided in Sect. IV. The novel topology is then adopted to design a $15-\mathrm{GHz}$ divide-by-three prescaler for a frequency synthesizer in a $5 \mathrm{G}$ wireless radio (Sect. V), reaching a remarkable improvement of the locking range and divider figure of merit (FoM).

\section{INTERMIXING IN DIRECT-INJECTION ILFDS}

Aim of this section is to highlight the most relevant harmonic terms generated by input-output intermixing across the injectors in Fig. 1. The ILFD will be considered in locking conditions, with the input radial frequency $\omega_{\text {in }}=2 \pi f_{\text {in }}$, equal to three times the output radial frequency $\omega_{\text {osc }}=2 \pi f_{\text {osc }}$ (i.e. $\omega_{o s c}=\omega$ and $\omega_{i n}=3 \omega$ ). The input and output differential signals, taken as harmonic signals with different phases, can be written as

$$
\begin{aligned}
V_{\text {in }, d} & =V_{\text {in }}^{+}-V_{\text {in }}^{-}=2 A_{\text {inj }} \sin (3 \omega t+\gamma) \\
V_{\text {out }, d} & =V_{\text {out }}^{+}-V_{\text {out }}^{-}=2 A_{\text {out }} \sin (\omega t+\varphi)
\end{aligned}
$$

where the single-ended zero-peak amplitudes are denoted as $A_{i n j}$ and $A_{\text {out }}$, respectively.

The analysis is carried out in two steps. Calculations are first performed by considering the MOSFETs of the injectors as they were in ohmic region for the whole time, i.e. with an $I_{d s}$ current given by

$$
I_{d s}=K \cdot\left(2 V_{o v} V_{d s}-V_{d s}^{2}\right)
$$

where the constant $K$ is $(1 / 2) \mu C_{o x}^{\prime}(W / L), V_{o v}$ the overdrive voltage $\left(V_{g s}-V_{T}\right)$, and $V_{T}$ the threshold voltage. The analysis is then extended to the more realistic large-signal operation of the injectors.

\section{A. Floating-Source Injector (Ohmic Regime)}

Let us consider first the floating-source injector $\left(M_{1 a}\right.$ and $M_{1 b}$ in Fig. 1), whose currents $I_{1, a}$ and $I_{1, b}$ can be calculated relying on (3). During the positive swing of the output voltage, the source terminals of $M_{1 a}$ and $M_{1 b}$ are oriented as in Fig. 1, so the overdrive voltages of the two MOSFETs are written as

$$
\begin{aligned}
V_{o v, 1 a}= & V_{i n, d c}+A_{\text {inj }} \sin (3 \omega t+\gamma)-V_{c m}-V_{T} \\
V_{o v, 1 b}= & V_{\text {in }, d c}-A_{\text {inj }} \sin (3 \omega t+\gamma)-V_{\text {out }, d c} \\
& +A_{\text {out }} \sin (\omega t+\varphi)-V_{T}
\end{aligned}
$$

where $V_{\text {in,dc }}$ and $V_{\text {out }, d c}$ are the DC bias values of the input and output nodes, and $V_{c m}$ denotes the potential of the common node between $M_{1 a}$ and $M_{1 b}$. In a similar way, the drain-source voltages are given by

$$
\begin{aligned}
& V_{d s, 1 a}=V_{\text {out }, d c}+A_{\text {out }} \sin (\omega t+\varphi)-V_{c m} \\
& V_{d s, 1 b}=V_{\text {cm }}-V_{\text {out }, d c}+A_{\text {out }} \sin (\omega t+\varphi) .
\end{aligned}
$$

The voltage $V_{c m}$ at the common node is derived, as in [7], by equating the two injectors' current $\left(I_{1 a}=I_{1 b}\right)$, using the model in (3), and neglecting the term $\left(V_{c m}-V_{o u t, d c}\right)^{2}$. The latter simplification corresponds to assume small signals across the injector, i.e. $A_{o u t}, A_{i n j}<<V_{o v}$. The resulting expression of $V_{c m}$ is therefore given by

$$
\begin{aligned}
V_{c m}= & {\left[V_{\text {out }, d c}-\frac{A_{o u t}^{2}}{4 V_{o v}, d c}\right] } \\
& +\frac{A_{o u t}^{2}}{4 V_{o v, d c}} \cos (2 \omega t+2 \varphi) \\
& +\frac{A_{\text {inj }} A_{\text {out }}}{2 V_{o v, d c}} \cos (2 \omega t+\gamma-\varphi)+ \\
& -\frac{A_{\text {inj }} A_{o u t}}{2 V_{o v, d c}} \cos (4 \omega t+\gamma+\varphi)
\end{aligned}
$$

where $V_{o v, d c}=V_{\text {in }, d c}-V_{o u t, d c}-V_{T}$.

To make the reading easier, let us introduce a more compact notation highlighting the different frequency components of the various signals. For example, (8) will be rewritten as follows

$$
V_{c m}=V_{c m}^{(0)}+V_{c m}^{\left(2^{\circ}\right)}+V_{c m}^{\left(2^{\prime}\right)}+V_{c m}^{\left(4^{\prime}\right)}
$$

where $V_{c m}^{(0)}$ is the DC term, while for the harmonics, the number in the superscript refers to the harmonic order, the apex (') highlights that the term is dependent on the input signal phase, $\gamma$, and the apex $\left(^{\circ}\right)$ means that the term is independent on $\gamma$. In (9), $V_{c m}^{(0)}$ and $V_{c m}^{\left(2^{\circ}\right)}$ do not depend on $\gamma$.

In a similar way, relying on (9), the harmonic expansion of $V_{o v, 1 a}$ in (4) can be written as

$$
V_{o v, 1 a}=V_{o v, 1 a}^{(0)}+V_{o v, 1 a}^{\left(3^{\prime}\right)}-V_{c m}^{\left(2^{\circ}\right)}-V_{c m}^{\left(2^{\prime}\right)}-V_{c m}^{\left(4^{\prime}\right)}
$$

including all the DC terms in $V_{o v}^{(0)}, 1 a$. Sorting the terms on the basis of the harmonic indexes, it can be rewritten as

$$
V_{o v, 1 a}=V_{o v, 1 a}^{(0)}-V_{c m}^{\left(2^{\circ}\right)}-V_{c m}^{\left(2^{\prime}\right)}+V_{o v, 1 a}^{\left(3^{\prime}\right)}-V_{c m}^{\left(4^{\prime}\right)} .
$$

Following the same procedure, (6) is rewritten as

$$
V_{d s, 1 a}=V_{d s, 1 a}^{(0)}+V_{d s, 1 a}^{\left(1^{\circ}\right)}-V_{c m}^{\left(2^{\circ}\right)}-V_{c m}^{\left(2^{\prime}\right)}-V_{c m}^{\left(4^{\prime}\right)} .
$$

The harmonics of the injector current $I_{1 a}$ can be computed combining (3), (11) and (12). Limiting our analysis to the first harmonic, $I_{1 a}^{(1)}$, the relevant intermixing terms are those listed in the table in Fig. 2. They can be further divided into two groups, i.e. those retaining the phase $\gamma$ of the input signal (highlighted by boxes in Fig. 2), and those independent of $\gamma$. Thus, using our notation, the first-order harmonic of $I_{1 a}$ is correspondingly written as

$$
I_{1 a}^{(1)}=I_{1 a}^{\left(1^{\prime}\right)}+I_{1 a}^{\left(1^{\circ}\right)}
$$




\begin{tabular}{|c|c|}
\hline $2 \mathrm{~V}_{\mathrm{ov}, 1 \mathrm{a}} \mathrm{V}_{\mathrm{ds}, 1 \mathrm{a}}$ & $-\mathbf{V}_{\mathrm{ds}, 1 \mathrm{a}}^{2}$ \\
\hline $2 V_{o v, 1 a}^{(0)} V_{d s, 1 a}^{\left(1^{\circ}\right)}$ & $-2 V_{d s, 1 a}^{(0)} V_{d s, 1 a}^{\left(1^{\circ}\right)}$ \\
\hline$-2 V_{c m}^{\left(2^{\prime}\right)} V_{d s, 1 a}^{\left(1^{\circ}\right)}$ & $2 V_{c m}^{\left(2^{\prime}\right)} V_{d s, 1 a}^{\left(1^{\circ}\right)}$ \\
\hline$-2 V_{c m}^{\left(2^{\circ}\right)} V_{d s, 1 a}^{\left(1^{\circ}\right)}$ & $2 V_{c m}^{\left(2^{\circ}\right)} V_{d s, 1 a}^{\left(1^{\circ}\right)}$ \\
\hline$-2 V_{o v, 1 a}^{\left(3^{\prime}\right)} V_{c m}^{\left(2^{\prime}\right)}$ & \\
\hline$-2 V_{o v, 1 a}^{\left(3^{\prime}\right)} V_{c m}^{\left(2^{\circ}\right)}$ & \\
\hline$-2 V_{o v, 1 a}^{\left(3^{\prime}\right)} V_{c m}^{\left(4^{\prime}\right)}$ & \\
\hline
\end{tabular}

Fig. 2. Beating terms generating the harmonic component of the $M_{1 a}$ current at the ILFD output frequency. The terms highlighted by boxes are the only ones contributing to the first harmonic of the injector current component dependent on the phase $\gamma$ of the input signal.

where $I_{1 a}^{\left(1^{\prime}\right)}$ contributes to the injection current, being dependent on $\gamma$, while $I_{1 a}^{\left(1^{\circ}\right)}$ is a resistive current independent of the input signal, in phase with $V_{\text {out }, d}$. From Fig. 2, it is easy to verify that the only term contributing to $I_{1 a}^{\left(1^{\prime}\right)}$ is the one from $-2 V_{o v, 1 a}^{\left(3^{\prime}\right)} V_{c m}^{\left(2^{\circ}\right)}$. Thus, the effective injection current $I_{i n j, 1}$, with the direction defined in Fig. 1 is given by

$$
I_{i n j, 1}=-I_{1 a}^{\left(1^{\prime}\right)}=A_{I, i n j, 1} \sin (\omega t+\gamma-2 \varphi)
$$

where

$$
A_{I, i n j, 1}=K \frac{A_{i n j} A_{o u t}^{2}}{4 V_{o v, d c}} .
$$

Without loss of generality, we can assume that the current $I_{o s c}$ delivered by the cross-coupled pairs $\left(M_{3 a}, M_{3 b}\right)$ and $\left(M_{3 c}, M_{3 d}\right)$ in Fig. 1 follows the phase of $V_{\text {out }, d}$ (i.e. the transconductor introduces negligible delay). Thus, from (2),

$$
I_{o s c}=A_{I, o s c} \sin (\omega t+\varphi) .
$$

If $\omega$ is exactly the resonance frequency of the LC tank, the oscillation current $I_{O S C}$ and the injection current $I_{i n j, 1}$ must have the same phase. Otherwise, the resulting tank current would be delayed with respect to $V_{\text {out }, d}$ and the oscillation frequency would depart from resonance [8]. As a result, combining (16) and (14), the following condition holds:

$$
\gamma-2 \varphi=\varphi \rightarrow \gamma=3 \varphi .
$$

The condition means that, at resonance, the leading edges of the two waveforms in (1) and (2) start synchronously, as it can be readily verified by circuit simulations.

\section{B. Floating-Source Injector (Large-Signal Regime)}

As the injectors are fully switched by large driving signals, we may wonder if the simplifications made so far are valid. To this purpose, we will now extend the previous results to large-signal regime and demonstrate that the main conclusions derived from the ohmic model still hold.
Since the injector circuit is symmetric and differentially driven by sine waveforms, the $V_{c m}$ signal is the sum of cosine functions at even harmonics. Thus, (8) can be put in a more general form as follows

$$
V_{c m}=V_{c m}^{(0)}+\sum_{n=1}^{\infty} V_{c m}^{\left(2 n^{\circ}\right)}+\sum_{n=1}^{\infty} V_{c m}^{\left(2 n^{\prime}\right)}
$$

where $V_{c m}^{\left(2 n^{\circ}\right)} \sim \cos (2 n \omega t+2 n \varphi)$ and $V_{c m}^{\left(2 n^{\prime}\right)} \sim \cos (2 n \omega t+$ $a \gamma+b \varphi)$ terms, with $a$ and $b$ relative integers.

In this frame, (4) and (6) are replaced by

$$
\begin{aligned}
V_{o v, 1 a}= & V_{o v, 1 a}^{(0)}+A_{i n j} \sin (3 \omega t+\gamma)+ \\
& -\sum_{n=1}^{\infty} V_{c m}^{\left(2 n^{\circ}\right)}-\sum_{n=1}^{\infty} V_{c m}^{\left(2 n^{\prime}\right)} \\
V_{d s, 1 a}= & V_{d s, 1 a}^{(0)}+A_{o u t} \sin (\omega t+\varphi)+ \\
& -\sum_{n=1}^{\infty} V_{c m}^{\left(2 n^{\circ}\right)}-\sum_{n=1}^{\infty} V_{c m}^{\left(2 n^{\prime}\right)}
\end{aligned}
$$

As a final step, to be more general, the current flowing through $M_{1 a}$ may be written as

$$
I_{1 a}=G\left(V_{o v, 1 a}, V_{d s, 1 a}\right) \cdot V_{d s, 1 a}
$$

where a non-linear conductance $G\left(V_{o v}, 1 a, V_{d s, 1 a}\right)$ links the MOSFET current to $V_{d s, 1 a}$. To derive the harmonic components of $I_{1 a}, G\left(V_{o v, 1 a}, V_{d s, 1 a}\right)$ is expanded in Taylor's series

$$
\begin{aligned}
& G\left(V_{o v, 1 a}, V_{d s, 1 a}\right) \\
& \approx G_{0}+G_{x}^{\prime} V_{o v, 1 a}+G_{y}^{\prime} V_{d s, 1 a} \\
& \quad+G_{x x}^{\prime} V_{o v, 1 a}^{2}+G_{x y}^{\prime} V_{o v, 1 a} V_{d s, 1 a}+G_{y y}^{\prime} V_{d s, 1 a}^{2}+\ldots
\end{aligned}
$$

and inserted into (21), together with (19) and (20). Let's now focus on the beat tones at $\omega$, generated by the intermixing terms $V_{o v, 1 a}^{n} V_{d s, 1 a}^{m}$ and contributing to the component $I_{1 a}^{(1)}$. To this aim, note that: i) a beat tone at an odd frequency is generated by intermixing an odd number of harmonics at odd frequencies (e.g. $4 \omega-2 \omega-\omega=\omega$ ); ii) the harmonics at odd frequencies in (19) and (20) are sine functions, while those at even frequencies are cosines. It follows that, since all intermixing terms contributing to $I_{1 a}^{(1)}$ result from the product of an odd number of sine functions, they are sine functions. Moreover, as in (13), $I_{1 a}^{(1)}$ can be split into a resistive term, $I_{1 a}^{\left(1^{\circ}\right)}$, following $V_{\text {out }, d} \sim \sin (\omega t+\varphi)$, and an injection current given by tones at $\omega$ fulfilling the intermixing condition

$$
h \cdot 3 \omega t-k \cdot \omega t= \pm \omega t \rightarrow k=3 h \mp 1 .
$$

In the most general case, the effective injection current, $I_{i n j, 1}$ may be written as

$$
\begin{aligned}
I_{i n j, 1}=\sum_{h=1}^{\infty} A_{i n j, 1, h} \sin [\omega t+h \gamma-(3 h-1) \varphi] & \\
& +\sum_{h=1}^{\infty} B_{i n j, 1, h} \sin [\omega t+(3 h+1) \varphi-h \gamma]
\end{aligned}
$$

Note that the simpler (14) corresponds to the first term of (24). Despite the presence of more terms in (24), all 


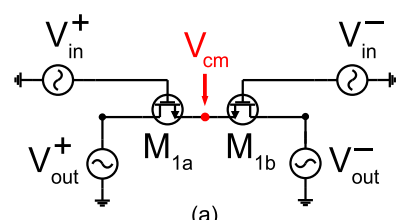

(a)
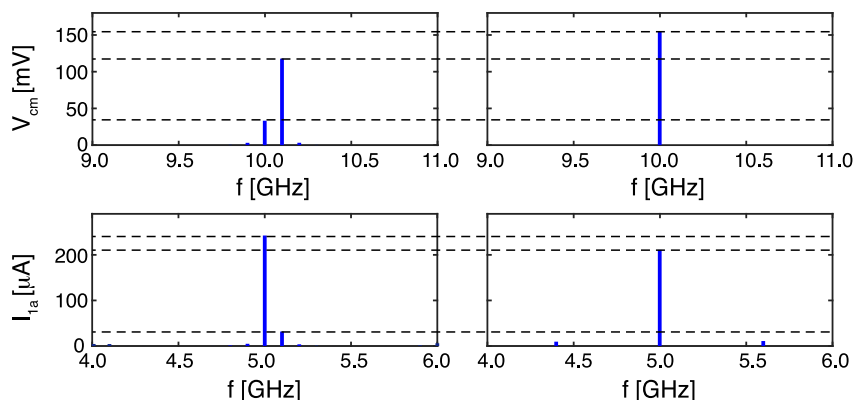

(b)

(c)

Fig. 3. Spectra of the common-mode voltage, $V_{c m}$, and of the floating-source injector current $I_{1 a}$. The circuit in (a) shows the simulation setup. The DC bias, $V_{\text {out }, d c}$, of both the output nodes and of the body terminals is $450 \mathrm{mV}$. The DC bias of the input nodes is $V_{i n, d c}=700 \mathrm{mV}$. The output nodes are driven by harmonic signals at $f_{\text {out }}=5 \mathrm{GHz}$, with opposite phases and a zero-peak voltage amplitude $A_{\text {out }}=200 \mathrm{mV}$. The input signals are also harmonic with an amplitude $A_{i n j}=300 \mathrm{mV}$, opposite phases and: (b) $f_{\text {in }}=15.1 \mathrm{GHz}$, (c) $f_{\text {in }}=15 \mathrm{GHz}, \gamma=3 \varphi$. The transistors' form factors are $(W / L)_{1 a}=(W / L)_{1 b}=(20 \mu \mathrm{m} / 0.06 \mu \mathrm{m})$.

of them become synchronous with $(\omega t+\varphi)$ for $\gamma=3 \varphi$, as in the ohmic-regime approximation. The actual orientation of $I_{i n j, 1}$ depends instead on the magnitude and the signs of $A_{i n j, 1, h}, B_{i n j, 1, h}$. However, from a practical standpoint, since the amplitude of the beat tones quickly vanishes by increasing its order, the injection current is expected to retain the same orientation of (14). To clearly assess the orientation of $I_{i n j, 1}$, circuit simulations of the real floating-source injector were performed using the simulation set-up in Fig. 3(a). Two sinusoids $\left(V_{i n}^{+}\right.$and $V_{i n}^{-}$) with $A_{i n j}=300 \mathrm{mV}$ zero-peak amplitude drive in anti-phase the gate terminals of $M_{1 a}$ and $M_{1 b}$, at frequency $f_{i n}$, while two sinuoids $\left(V_{\text {out }}^{+}\right.$and $\left.V_{\text {out }}^{-}\right)$ with $A_{\text {out }}=200 \mathrm{mV}$ at $f_{\text {osc }}=5 \mathrm{GHz}$ drive in anti-phase the output terminals of the injector. The spectra in Fig. 3(b) refers to $f_{\text {in }}=15.1 \mathrm{GHz}$, where the $100-\mathrm{MHz}$ offset between $f_{\text {in }}$ and $3 f_{\text {osc }}$ makes it possible to identify the signal components generated by intermixing with the input signal. In fact, the spectrum of $V_{c m}$ in Fig. 3(b) shows a tone at $2 f_{\text {osc }}=$ $10.0 \mathrm{GHz}$ [which is essentially due to $V_{c m}^{\left(2^{\circ}\right)}$ in (9)], and a tone at $f_{\text {in }}-f_{\text {osc }}=10.1 \mathrm{GHz}$, which is due to intermixing with the input signal [term $V_{c m}^{\left(2^{\prime}\right)}$ in (9)]. The injector current has a tone at $f_{\text {osc }}=5.0 \mathrm{GHz}$, resulting from resistive conduction, while intermixing generates the dominant component at $f_{\text {in }}-2 f_{\text {osc }}=5.1 \mathrm{GHz}$ in (14). The other higher order beat tones at $[\ldots, 4.8,4.9,5.2, \ldots] \mathrm{GHz}$ are hardly visible. To check for the relative phases, the simulation was repeated by shifting the input signal frequency to $f_{i n}=15 \mathrm{GHz}$ and taking $\gamma=3 \varphi$, thus making the harmonics overlapping. The results are in Fig. 3(c). Note that the $V_{c m}$ amplitude increases, thus confirming that the second-harmonic terms in (8) add up with phase coherence. The current tone at $5 \mathrm{GHz}$ decreases, instead, meaning that the injection current is opposite to $V_{\text {out }}$, and to the resistive current, $I_{1 a}^{\left(1^{\circ}\right)}$, in agreement with the sign in (14).

\section{Divide-By-Two Locking}

The concept behind the ILFD by three with divide-by-two locking [2] is to use the $V_{c m}$ voltage tone at $f_{i n}-f_{\text {osc }}$ to drive the additional direct injector $M_{2}$. Figure 1 shows the resulting circuit and the additional injection current, $I_{i n j, 2}$.

To compute this term, let us apply the ohmic MOSFET model to $M_{2}$, thus writing

$$
I_{2}=K_{2}\left(2 V_{o v, 2} V_{d s, 2}-V_{d s, 2}^{2}\right) .
$$

Note that, due to the capacitive coupling between the central node of the floating-source injector and the gate of $M_{2}$, the DC term of $V_{c m}$ (i.e. $\left.V_{c m}^{(0)}\right)$ does not contribute to the $M_{2}$ gate signal, $V_{g, 2}$. Therefore, we may write

$$
V_{g, 2}=V_{b 2}+\left(V_{c m}-V_{c m}^{(0)}\right)
$$

leading to the $M_{2}$ overdrive voltage

$$
\begin{aligned}
V_{o v, 2}=V_{b 2}+\left(V_{c m}\right. & \left.-V_{c m}^{(0)}\right)+ \\
& -V_{\text {out }, d c}+A_{\text {out }} \sin (\omega t+\varphi)-V_{T}
\end{aligned}
$$

and to

$$
\begin{aligned}
V_{\text {ov }, 2}= & V_{b 2}+V_{c m}^{\left(2^{\circ}\right)}+V_{c m}^{\left(2^{\prime}\right)}+V_{c m}^{\left(4^{\prime}\right)}+ \\
& -V_{\text {out }, d c}+A_{\text {out }} \sin (\omega t+\varphi)-V_{T} \\
V_{d s, 2}= & 2 A_{\text {out }} \sin (\omega t+\varphi) .
\end{aligned}
$$

Following the compact notation introduced before, we may order the harmonics and list them as

$$
\begin{aligned}
& V_{o v, 2}=V_{o v, 2}^{(0)}+V_{o v, 2}^{\left(1^{\circ}\right)}+V_{c m}^{\left(2^{\circ}\right)}+V_{c m}^{\left(2^{\prime}\right)}+V_{c m}^{\left(4^{\prime}\right)} \\
& V_{d s, 2}=V_{d s, 2}^{\left(1^{\circ}\right)} .
\end{aligned}
$$

Also in this case, the first harmonic $I_{2}^{(1)}$ of the total current $I_{2}$ of $M_{2}$ can be written as

$$
I_{2}^{(1)}=I_{2}^{\left(1^{\prime}\right)}+I_{2}^{\left(1^{\circ}\right)} .
$$

From the products in (25), it turns out that $I_{2}^{(1)}$ is generated by the following terms:

$$
2 K_{2} V_{o v, 2}^{(0)} V_{d s, 2}^{\left(1^{\circ}\right)} ; \quad 2 K_{2} V_{c m}^{\left(2^{\circ}\right)} V_{d s, 2}^{\left(1^{\circ}\right)} ; \quad 2 K_{2} V_{c m}^{\left(2^{\prime}\right)} V_{d s, 2}^{\left(1^{\circ}\right)}
$$

where the only term contributing to the injection current is due to $2 K_{2} V_{c m}^{\left(2^{\prime}\right)} V_{d s, 2}^{\left(1^{\circ}\right)}$, which gives a down-converted component depending on the phase $\gamma$ of the input signal. It turns out that the effective injection current $I_{i n j, 2}$ of the second injector is

$$
I_{i n j, 2}=-I_{2}^{\left(1^{\prime}\right)}=A_{I, i n j, 2} \sin (\omega t+\gamma-2 \varphi)
$$

where

$$
A_{I, i n j, 2}=K_{2} \frac{A_{i n j} A_{o u t}^{2}}{V_{o v, d c}} .
$$

Also in this case, a large-signal analysis similar to the previous ones can be carried out. To check for large-signal operation, simulations were performed on the circuit in Fig. 4(a). Each output node is driven by a $A_{\text {out }}=200 \mathrm{mV}$ zero-topeak amplitude. The input voltage, $V_{i n}$, driving the $M_{2}$ gate, 

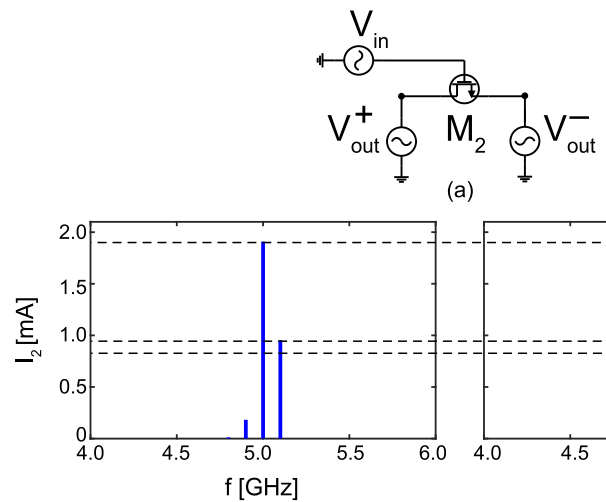

(b)

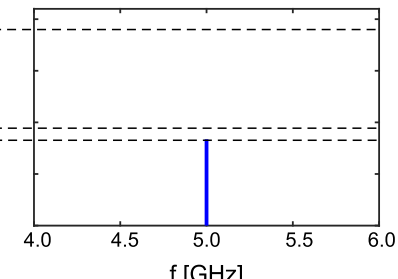

(c)

Fig. 4. Spectrum of the current $I_{2}$ flowing through the $M_{2}$ injector. The circuit in (a) shows the simulation setup. The DC bias, $V_{\text {out }}, d c$, of both the output nodes and of the body terminals is $450 \mathrm{mV}$. The DC bias of the input nodes is $V_{i n, d c}=700 \mathrm{mV}$. The output nodes are driven by harmonic signals at $f_{\text {out }}=5 \mathrm{GHz}$, with opposite phases and a zero-peak voltage amplitude $A_{\text {out }}=200 \mathrm{mV}$. The input signal is also harmonic with a zero-peak voltage amplitude of $100 \mathrm{mV}$ and a frequency: (b) $f_{\text {in }}=10.1 \mathrm{GHz}$, (c) $f_{\text {in }}=10 \mathrm{GHz}$. The transistor form factor is $(W / L)_{2}=(60 \mu \mathrm{m} / 0.06 \mu \mathrm{m})$.

has instead a 100-mV zero-peak amplitude. To be consistent with the relative phase, a $90^{\circ}$ phase shift is kept between $V_{i n}$, which is a cosine [see $V_{c m}^{\left(2^{\prime}\right)}$ in (8)], and $V_{\text {out }}$ [see $V_{\text {out }, d}$ in (2)]. The first simulations were performed by using an input signal frequency $f_{\text {in }}=10.1 \mathrm{GHz}$, to separate the different current components [Fig. 4(b)]. Then, the simulations were repeated with $f_{\text {in }}=10 \mathrm{GHz}$, aligning the harmonic signals with $\gamma=3 \varphi$, to verify the relative sign of the different terms [Fig. 4(c)]. Note that the amplitude of the tone at $5 \mathrm{GHz}$ in Fig. 4(c) is equal to the difference between the amplitudes of $I_{2}^{\left(1^{\circ}\right)}$ at $5 \mathrm{GHz}$ and $I_{2}^{\left(1^{\prime}\right)}$ at $5.1 \mathrm{GHz}$ in Fig. 4(b). The effective injection current, $I_{i n j, 2}$ is therefore positive in the direction in Fig. 1, as predicted from the sign in (34).

\section{NOVEL ILFD TOPOLOGY}

The analysis proposed above brings to a key observation. The down-converted voltage tone, $V_{c m}^{\left(2^{\prime}\right)}$, can be also exploited to drive the gate terminal of the tail transistor $M_{4}$ (Fig. 5), thus generating an additional current component, $I_{i n j, 3}$, which can reinforce the injection. The injected current $I_{i n j, 3}$ can be computed by calculating $I_{3 a}^{\left(1^{\prime}\right)}$, i.e. the component of the first harmonic of the current in $M_{3 a}$ that is dependent on the input phase $\gamma$.

For small $V_{c m}$ signals, the tail current signal in $M_{4}$ can be written as

$$
\begin{aligned}
I_{4} & =I_{d c}-g_{m, 4}\left(V_{c m}^{\left(2^{\circ}\right)}+V_{c m}^{\left(2^{\prime}\right)}+V_{c m}^{\left(4^{\prime}\right)}\right) \\
& =I_{4}^{(0)}+I_{4}^{\left(2^{\circ}\right)}+I_{4}^{\left(2^{\prime}\right)}+I_{4}^{\left(4^{\prime}\right)} .
\end{aligned}
$$

The fundamental harmonic of the mixing transfer function due to the cross-coupled pairs oscillation is

$$
X_{c c p}^{\left(1^{\circ}\right)}=\frac{4}{\pi} \sin (\omega t+\varphi) \text {. }
$$

The harmonics of the current, $I_{4}$, beat with $X_{c c p}^{\left(1^{\circ}\right)}$, leading to harmonics of the current $I_{3 a}$, that flows via $\left(M_{3 a}, M_{3 b}\right)$ to

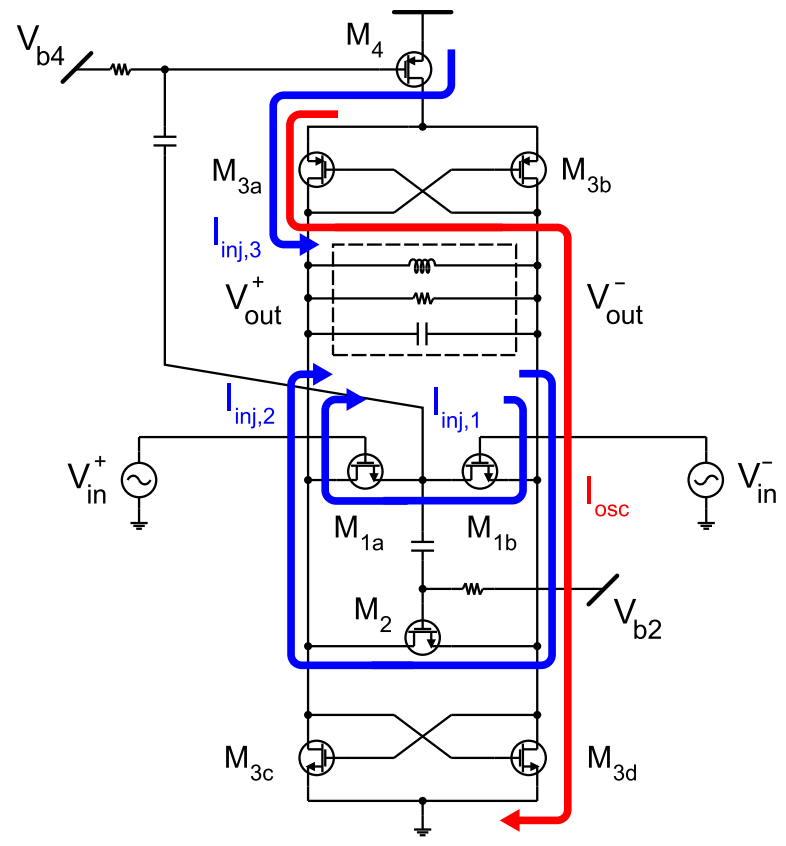

Fig. 5. Novel ILFD by three with dual-injection divide-by-two locking. The arrows denote the injected currents $I_{i n j, 1}, I_{i n j, 2}, I_{i n j, 3}$, and the oscillation current $I_{o s c}$. The positions of the source terminals of $M_{1 a}, M_{1 b}$ and $M_{2}$ refer to the case of a positive output differential voltage $\left(V_{\text {out }}^{+}-V_{\text {out }}^{-}\right)$.

the tank. The first harmonic component, $I_{3 a}^{(1)}$, is generated by the following intermixing products:

$$
I_{4}^{(0)} X_{c c p}^{\left(1^{\circ}\right)} ; \quad I_{4}^{\left(2^{\circ}\right)} X_{c c p}^{\left(1^{\circ}\right)} ; \quad I_{4}^{\left(2^{\prime}\right)} X_{c c p}^{\left(1^{\circ}\right)}
$$

where the only term depending on the phase of the input signal, $\gamma$, is the last one:

$I_{4}^{\left(2^{\prime}\right)} X_{c c p}^{\left(1^{\circ}\right)}=-g_{m, 4} \frac{A_{i n j} A_{o u t}}{2 V_{o v, d c}} \cos (2 \omega t+\gamma-\varphi) \frac{4}{\pi} \sin (\omega t+\varphi)$.

The expression of the effective injection current $I_{i n j, 3}$ follows directly from (39) taking the beat tone at $\omega$

$$
I_{i n j, 3}=I_{3 a}^{\left(1^{\prime}\right)}=A_{I, i n j, 3} \sin (\omega t+\gamma-2 \varphi)
$$

where

$$
A_{I, i n j, 3}=\frac{g_{m, 4}}{\pi} \frac{A_{i n j} A_{o u t}}{V_{o v, d c}} \sin (\omega t+\gamma-2 \varphi) .
$$

The analysis shows that the tail path gives rise to an injection current which flows out of $M_{3 a}$ with the same orientation of $I_{o s c}$, and adds in phase to $I_{i n j, 1}$ and $I_{i n j, 2}$, reinforcing the injection-locking mechanism.

Following the same approach used above, the result was checked by simulating the circuit in Fig. 6(a). The input and output voltage amplitudes were the same as in the analysis of the direct-injector $M_{2}$, with a $90^{\circ}$ phase shift between the signals. Two simulations were performed, with an input frequency $f_{\text {in }}$ of $10.1 \mathrm{GHz}$ and $10 \mathrm{GHz}$, respectively. Fig. 6(b) and (c) show the resulting spectra. Note that the sum of the amplitudes of the 5-GHz tone, which is in phase with $V_{\text {out }, d}$, and the 5.1-GHz injection tone in Fig. 6(b) is equal to the 

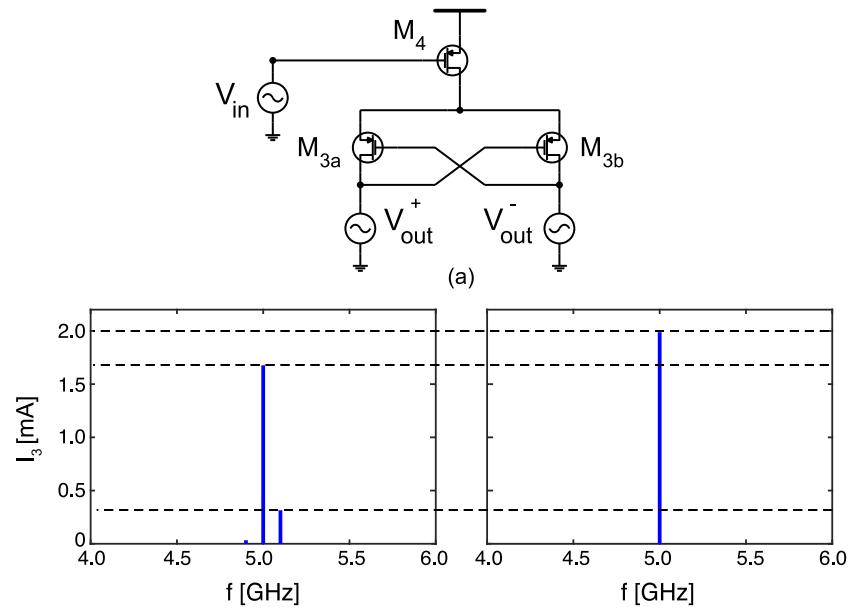

(b)

(c)

Fig. 6. Spectrum of the current $I_{3}$, flowing via $M_{3 a}$ and $M_{3 b}$ to the tank. The circuit in (a) shows the simulation setup. The DC bias of the output nodes is $V_{\text {out }, d c}=450 \mathrm{mV}$, while the DC bias of the input node is $V_{i n, d c}=550 \mathrm{mV}$ The output nodes are driven by harmonic signals at $f_{\text {out }}=5 \mathrm{GHz}$, with opposite phases and a zero-peak voltage amplitude $A_{\text {out }}=200 \mathrm{mV}$. The input signal is also harmonic with a zero-peak voltage amplitude of $100 \mathrm{mV}$ and a frequency: (b) $f_{\text {in }}=10.1 \mathrm{GHz}$, (c) $f_{\text {in }}=10 \mathrm{GHz}$. The transistors' form factors are $(W / L)_{4}=(56 \mu \mathrm{m} / 0.06 \mu \mathrm{m})$ and $(W / L)_{3}=(69 \mu \mathrm{m} / 0.06 \mu \mathrm{m})$.

amplitude of the tone at $5 \mathrm{GHz}$ in Fig. 6(c), in agreement with the analysis.

In the novel circuit, the overall injection current flowing through the tank is given by the coherent superposition of all the three terms, leading to a higher total injection current and, therefore, to a wider locking range [8]:

$$
\Delta \omega_{\max } \approx \frac{\omega_{0}}{2 Q_{t o t}} \frac{I_{i n j, 1}+I_{i n j, 2}+I_{i n j, 3}}{I_{o s c}}
$$

where $Q_{t o t}$ is the tank quality factor, when loaded by the injectors, and $\omega_{0}=2 \pi f_{0}$ is the resonance radial frequency. The first term at the RHS of (42) arises from the injection through the floating-source injector and corresponds to the locking range of the conventional ILFD by three. The second and third terms are generated by the divide-by-two paths through $M_{2}$ and $M_{4}$, respectively. Because of the presence of the third contribution, the proposed ILFD is therefore expected to feature a wider locking range, not only with respect to the conventional divide-by-three ILFD, but also compared to the ILFD with divide-by-two locking in Fig. 1, for the same power dissipation.

\section{Performance Comparison}

To quantitatively assess the potentials of the new topology, the schematic-level performance of the three single-inductor ILFD structures were compared, namely the conventional divide-by-three ILFD with floating-source injector, the divideby-three ILFD with divide-by-two locking and the proposed ILFD. Each ILFD circuit was designed in a 65-nm LP CMOS technology, and, to perform a fair comparison, separately optimized to meet the same performance: (i) a free-running oscillation frequency of $5 \mathrm{GHz}$ (i.e. the center frequency of the input locking range is $15 \mathrm{GHz}$ ), (ii) a DC current consumption of $2.2 \mathrm{~mA}$, (corresponding to a DC power consumption of
$2.64 \mathrm{~mW}$ for a $1.2 \mathrm{~V}$ voltage supply) and (iii) an output single-ended voltage amplitude, $A_{\text {out }}$, larger than $0.2 \mathrm{~V}$.

The ILFDs were designed with complementary cross-coupled pairs to halve the DC power consumption of the circuit for the same oscillation amplitude, keeping the output voltage swing within the supply voltage rails, thus improving the circuit reliability. In all the three ILFDs, the tail current generators were implemented with p-type MOSFETs. Low-threshold-voltage devices were used for all the transistors. In this way, the cross-coupled pairs have enough voltage headroom despite the three stacked transistors, and the injectors have reduced switching times and higher current capacity. Further reduction of their threshold voltage was obtained by tying the body terminals to the center tap of the tank inductor, thus preventing body effect [9].

The target oscillation amplitude for a given power consumption sets the minimum load parallel resistance across the tank to $R_{t o t} \approx 200 \Omega$. The latter value is given by the shunt composition of the injector resistance, $R_{i n j}$, and of the resistance, $R_{L}$, accounting for the intrinsic tank losses.

To boost the injected currents, the injectors' width must be maximized, thus reducing $R_{i n j}$, though keeping the $R_{t o t}$ requirement. Therefore, to leave room to injectors' conductivity, the $R_{L}$ value must be maximized. Since $R_{L}=\omega_{0} L Q_{L}$, this, in turn, calls for a large inductance, $L$. In practice, the inductor value was chosen as $L=4.1 \mathrm{nH}$ with a quality factor $Q_{L}=20$, corresponding to $R_{L} \approx 2.5 \mathrm{k} \Omega$ at the $5-\mathrm{GHz}$ resonance frequency.

In the conventional ILFD by three, the size of the injector was decided by sweeping the form factors of $M_{1 a}$ and $M_{1 b}$, from $(W / L)_{1}=(80 \mu \mathrm{m} / 0.06 \mu \mathrm{m})=1333$ to $(96 \mu \mathrm{m} / 0.06 \mu \mathrm{m})=1600$, and their gate bias, $V_{b 1}$, from $550 \mathrm{mV}$ to $850 \mathrm{mV}$. By increasing $V_{b 1}$, the locking range increases since the MOSFETs are on for a longer time interval, thus resulting in a higher injected current. Simulations show that the maximum locking range is obtained for $V_{b 1}$ ranging from $650 \mathrm{mV}$ to $750 \mathrm{mV}$, at the cost of a tolerable reduction of the output amplitude. Beyond this point, the locking range does not increase further, while the output voltage amplitude is significantly degraded. Based on these results, $V_{b 1}$ was set at $750 \mathrm{mV}$ and $(W / L)_{1}$ was set to $(88 \mu \mathrm{m} / 0.06 \mu \mathrm{m})=1466$ for both $M_{1 a}$ and $M_{1 b}$.

For the ILFD with divide-by-two locking in Fig. 1, the gate bias of the floating-source injector was kept to $V_{b 1}=750 \mathrm{mV}$, as above. To size the additional injector $M_{2}$, a similar optimization procedure was followed. The form factor of the floating-source injector was progressively reduced to leave room to lower the resistance of the second injector, in parallel to the tank, for the same target $R_{t o t}$. For each $(W / L)_{1}$ value, the dependence of the oscillation amplitude and the locking range vs. $(W / L)_{2}$ (i.e. the form factor of $\left.M_{2}\right)$ and $V_{b 2}$ were derived. The optimal values were identified as $(W / L)_{1}=$ $(20 \mu \mathrm{m} / 0.06 \mu \mathrm{m})=333$, and $(W / L)_{2}=(60 \mu \mathrm{m} / 0.06 \mu \mathrm{m})=$ 1000 for $V_{b 2}=650 \mathrm{mV}$.

Note that the optimal sizing is reached with $M_{2}$ three times bigger than $M_{1 a}$ and $M_{1 b}$. This outcome is in line with the results of the harmonic analysis. In fact, by comparing (15) and (35) it turns out that for the same $K$ value of the 


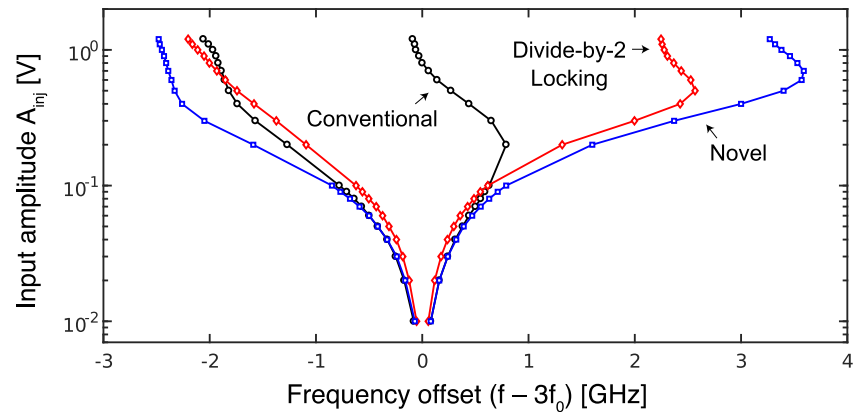

Fig. 7. Arnold tongues of the three ILFDs, separately optimized to meet the performance in Table I, simulated at the schematic level.

TABLE I

Simulated Performance in SAme 65-nm LP CMOS Process

\begin{tabular}{|c|c|c|c|}
\hline ILFD type & Conventional & $\begin{array}{c}\text { Divide-by-2 } \\
\text { locking }\end{array}$ & Proposed \\
\hline$V_{D D}(\mathrm{~V})$ & 1.2 & 1.2 & 1.2 \\
\hline$P_{\text {diss }}(\mathrm{mW})$ & 2.64 & 2.64 & 2.64 \\
\hline$A_{\text {out }}(\mathrm{V})^{\star}$ & 0.23 & 0.26 & 0.26 \\
\hline$f_{\text {center }}(\mathrm{GHz})$ & 15 & 15 & 15 \\
\hline Lock Range $(\%)^{\star \star}$ & 13 & 29 & 40 \\
\hline
\end{tabular}

injectors and the same bias and driving signals (i.e. $V_{o v}, d c$, $A_{i n j}$ and $\left.A_{\text {out }}\right)$ the injection current $I_{i n j, 2}$ generated by $M_{2}$ is larger than the injection current $I_{i n j, 1}$ generated by the floating-source injector. The observation suggests that to boost injection current and locking range, it is more convenient to increase $(W / L)_{2}$ rather than $(W / L)_{1}$, which is indeed what is derived by parametric optimization of the circuit.

Let us finally discuss the optimization of the novel ILFD (Fig. 5). Since the new injector $M_{4}$ is not added in parallel to the tank, it does not directly affect tank quality factor and ILFD oscillation amplitude. Therefore, the sizing and the bias of $M_{2}, M_{1 a}$ and $M_{1 b}$ were the same adopted for the ILFD with divide-by-two locking. The only difference was the addition of the AC coupling network linking the common source node and $M_{4}$ gate. Regarding $M_{4}$, a trade-off exists between the transconductance needed to boost injection current and the parasitic capacitance at the drain node, which diverts part of the modulated current to ground. The optimal sizing was found for a form factor of $(W / L)_{4}=(56 \mu \mathrm{m} / 0.06 \mu \mathrm{m})=933$ and $V_{b 4}=543 \mathrm{mV}$.

Figure 7 shows the Arnold tongues of the three circuits, while Table I lists their performance figures. For an input signal with $A_{i n j}=0.6 \mathrm{~V}$, the locking range values of the conventional ILFD, the ILFD with divide-by-two locking and the proposed ILFD are about 13\%, 29\% and $40 \%$ of the input central frequency $f_{\text {center }}=15 \mathrm{GHz}$, respectively, for the same power consumption. It follows that the novel topology can reach a locking range about three times larger than the conventional ILFD, and about a $40 \%$ improvement with respect to the ILFD with divide-by-two locking. Even at lower input amplitudes, (i.e. $A_{i n j}=0.4 \mathrm{~V}$ ), the improvement is still significant, i.e. about $31 \%$.

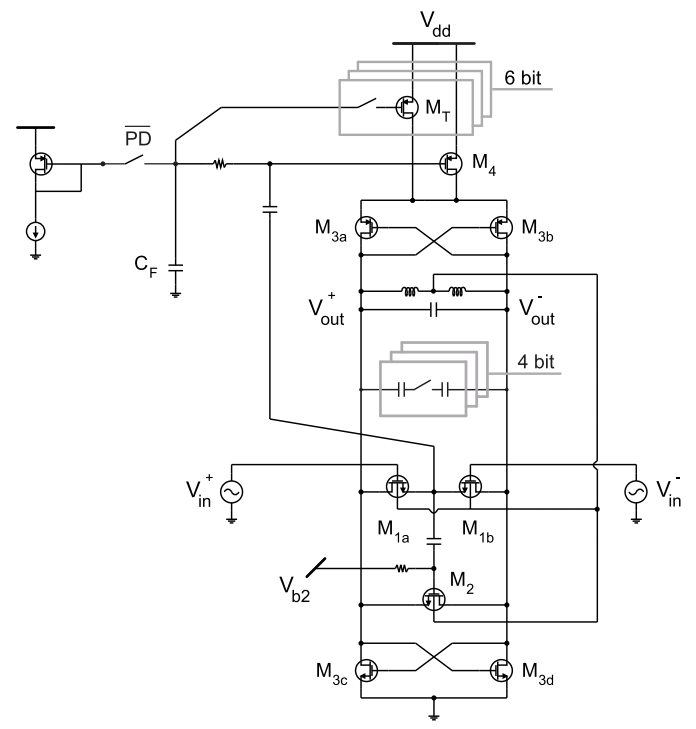

Fig. 8. Scheme of the single-inductor divide-by-three ILFD with dual-injection divide-by-two secondary locking designed in a $65 \mathrm{~nm}$ LP CMOS technology to work as $15 \mathrm{GHz}$ divider in a $5 \mathrm{G}$ frequency synthesizer.

Supply sensitivity has been verified superimposing a $100-\mathrm{kHz}$ tone with $100 \mu \mathrm{V}$ rms value to the $V_{d d}$. The three circuits exhibited almost the same sensitivity (i.e., a spur in the output spectrum of about $-83 \mathrm{dBc}$ at $100-\mathrm{kHz}$ offset), meaning that supply sensitivity is not affected by the topology modification.

\section{CiRCUit Design AND Simulations}

The novel topology was exploited to design a $15-\mathrm{GHz}$ divide-by-three ILFD for a frequency synthesizer targeting $5 \mathrm{G}$ radio specifications, with minimum DC power consumption $(1.5 \mathrm{~mW})$, a $4 \mathrm{GHz}$ locking range around $15 \mathrm{GHz}$, with an input voltage amplitude $A_{i n j}=0.4-0.45 \mathrm{~V}$ and a minimum output amplitude $A_{\text {out }}=0.2 \mathrm{~V}$ over the whole range, to guarantee good driving of the following stage. In the same low-cost $65 \mathrm{~nm}$ LP CMOS process, a divide-by-three CML stage would dissipate a power in excess of $10 \mathrm{~mW}$ with limited robustness.

To cover the target locking range while keeping low power consumption, the overall locking range was segmented by using a four-thermometric-bits capacitive bank in parallel to the tank. The capacitor bank was sized to guarantee more than $60 \%$ overlap between each single tuning interval. In this way, the frequency range was covered without gaps, even in presence of temperature and corner variations.

Additional transistors $\left(M_{T}\right.$ in Fig. 8), switchable with a six-bits thermometric coding, were added in parallel to $M_{4}$, to reach the target oscillation amplitude also on process corners, by properly changing the DC current value. Their gates are not modulated by $V_{c m}$, not to increase signal losses due to the higher parasitic capacitance in series to the AC coupling. They are instead connected to a fixed voltage bias, with a 6-pF $C_{F}$ capacitance to filter noise and disturbances coming from the external bias network. 
TABLE II

Comparison With the State of ART OF InJeCtion-Locked Frequency Dividers By ThreE

\begin{tabular}{|c|c|c|c|c|c|c|c|c|c|c|}
\hline Ref. & {$[4]^{\mathrm{a}}$} & {$[5]^{\mathrm{a}}$} & {$[10]^{\mathrm{a}}$} & {$[11]^{\mathrm{a}}$} & {$[12]^{\mathrm{a}}$} & {$[13]^{\mathrm{a}}$} & {$[14]^{\mathrm{a}}$} & {$[15]^{\mathrm{a}}$} & {$[2]^{\mathrm{a}}$} & This work $^{b}$ \\
\hline Tech. & $0.18 \mu \mathrm{m}$ & $0.13 \mu \mathrm{m}$ & $0.18 \mu \mathrm{m}$ & $0.18 \mu \mathrm{m}$ & $0.18 \mu \mathrm{m}$ & $0.18 \mu \mathrm{m}$ & $90 \mathrm{~nm}$ & $0.18 \mu \mathrm{m}$ & $0.18 \mu \mathrm{m}$ & $65 \mathrm{~nm}$ \\
\hline$\#$ of inductors & 5 & $5^{c}$ & 3 & 3 & 3 & 2 & 1 & 1 & 1 & 1 \\
\hline Area $\left(\mathrm{mm}^{2}\right)^{\mathrm{d}}$ & 0.81 & $0.83^{\mathrm{e}}$ & 0.67 & 0.43 & 0.53 & 0.47 & 0.14 & 0.22 & 0.14 & 0.09 \\
\hline$V_{D D}(\mathrm{~V})$ & 0.9 & 0.5 & 0.8 & 1.8 & 1.4 & 0.8 & 0.5 & 1.45 & 1.2 & 1.2 \\
\hline$P_{\text {diss }}(\mathrm{mW})$ & 6.76 & 2.05 & 7.72 & 3.90 & 5.60 & 4.90 & 2.85 & 5.13 & 4.20 & 1.56 \\
\hline$f_{\text {center }}(\mathrm{GHz})$ & 8.43 & 14.55 & 8.00 & 22.10 & 9.15 & 10.50 & 28.95 & 26.35 & 14.20 & 14.85 \\
\hline Lock Range (\%) & 84.2 & 35.0 & $50.0^{f}$ & $15.4^{\mathrm{f}}$ & 53.6 & 47.6 & $15.5^{\mathrm{f}}$ & 12.5 & 34.3 & 23.6 \\
\hline FoM $(\% / \mathrm{mW})$ & 12.5 & 17.1 & 6.5 & 3.9 & 9.6 & 9.7 & 5.4 & 2.4 & 8.2 & 15.1 \\
\hline $\begin{array}{l}\ddagger \text { All data refers } \\
\text { a Measured result } \\
\text { b Post-layout sim }\end{array}$ & & $00^{\circ}$ & & & $\begin{array}{l}{ }^{a} \text { Est } \\
\text { e } \text { Chi } \\
\text { f No }\end{array}$ & e. & & splici & & \\
\hline
\end{tabular}

${ }^{c}$ One inductor is implemented with external bondwire.

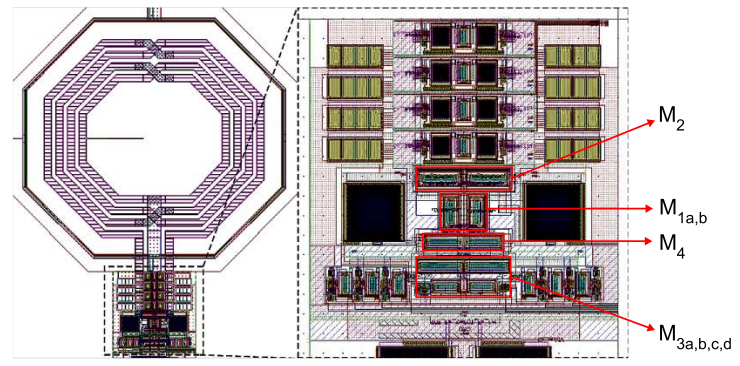

Fig. 9. Layout of the designed single-inductor ILFD (left) and zoomed view of the ILFD core (right).

Figure 9 shows the layout implementation of the designed ILFD. To minimize tank losses, the capacitor bank was placed as close as possible to the inductor. Since the $M_{2}$ injector has the largest injection strength, it is placed very close to the LC tank. The floating-source injector is beside it, and the tail transistor $M_{4}$ follows. With this choice, $M_{4}$ is located as close as possible to the p-type cross-coupled pairs $\left(M_{3 a}, M_{3 b}\right)$, thus minimizing the stray capacitance at its drain node.

The netlist was first extracted from the layout and then simulated with the PSS analysis of Cadence SpectreRF ${ }^{\circledR}$. To better account for the impact of the top metal levels on both the inductance and the quality factor values, the RC parasitics of the ILFD core were extracted with Mentor Graphics Calibre $^{\circledR}$ xRC, while the RLC parasitics of the coil and its interconnections were achieved using Keysight Momentum ${ }^{\circledR}$ electromagnetic simulator. The simulations were carried out on the post-layout extracted netlist, considering a $T=100^{\circ} \mathrm{C}$ temperature, which is the worst case for thermal noise and tank quality factor.

The plot in Fig. 10 shows the input sensitivity curves of the ILFD at the DC power of $1.56 \mathrm{~mW}$ as a function of the input frequency $f_{\text {in }}$. The five curves correspond to the possible settings of the capacitive bank. The widest locking range of $28 \%$ ( 12.9 to $17.1 \mathrm{GHz})$ is reached for $A_{i n j}=0.7 \mathrm{~V}$. With $A_{i n j}=0.3 \mathrm{~V}$, the locking range is $23.6 \%(13.1$ to $16.6 \mathrm{GHz})$, and, even with $A_{i n j}=0.15 \mathrm{~V}$, it still reaches a remarkable value of $19.7 \%$ (13.3 to $16.2 \mathrm{GHz}$ ).

Figure 11 shows the zero-peak amplitude of the single-ended output oscillation and the DC power, for $A_{i n j}=0.3 \mathrm{~V}$ input voltage amplitude. The output differential amplitude is about $0.44 \mathrm{~V}$, and it is rather constant along the

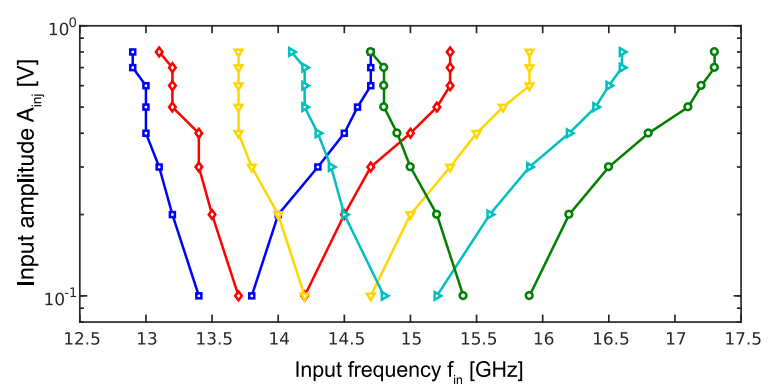

Fig. 10. Input sensitivity curves from post-layout simulations at $T=100^{\circ} \mathrm{C}$.
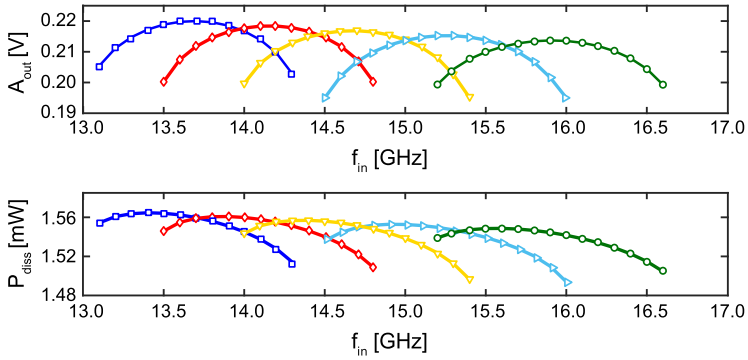

Fig. 11. Single-ended zero-peak output voltage amplitude, $A_{\text {out }}$, and DC power consumption with $A_{i n j}=0.3 \mathrm{~V}$, from post-layout simulation at $T=100^{\circ} \mathrm{C}$.

whole locking range, with proper setting of the capacitor bank. The same result holds for the dissipated power, which ranges between 1.52 and $1.56 \mathrm{~mW}$.

Figure 12 shows the phase noise at the output of the ILFD, with $A_{i n j}=0.3 \mathrm{~V}$ at $14.9 \mathrm{GHz}$, obtained from SpectreRF pnoise analysis. The white noise is $-143.5 \mathrm{dBc} / \mathrm{Hz}$, while the flicker has a corner frequency of $280 \mathrm{kHz}$. Finally, Table II compares the design figures with state-of-art divide-by-three ILFDs working with 0-dBm input power using the FoM values defined as in [10]

$$
\mathrm{FoM}=\frac{\mathrm{LR}_{(\%)}}{P_{\operatorname{diss}(m W)}} .
$$

Note that the proposed divider has the best FoM among those using a single inductor (last four columns in Table II) and features, in general, one of the highest FoM values among multi-inductor stages. To the best of our knowledge, only the divider proposed in [5] shows a similar FoM, but it needs five inductors instead than one. Thanks to the use of a single 


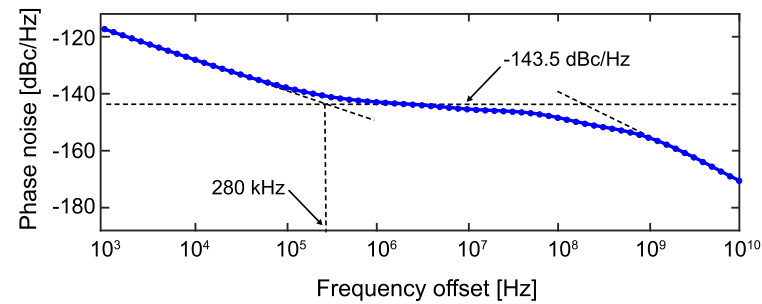

Fig. 12. Phase noise spectrum of the $4.96 \mathrm{GHz}$ output signal with $A_{i n j}=0.3 \mathrm{~V}$, from post-layout simulations at $T=100^{\circ} \mathrm{C}$.

inductor, the novel circuit occupies, instead, a very small area of only $332 \mu \mathrm{m}$ by $274 \mu \mathrm{m}$, that is $0.09 \mathrm{~mm}^{2}$.

\section{CONCLUSION}

A detailed analysis of the signal intermixing taking place across the injectors of the divider by three with divide-bytwo locking was presented, providing deeper insights in circuit operation and opening the way to the invention of a new divide-by-three ILFD. The latter widens by about $40 \%$ the typical locking range obtained with conventional divide-bytwo locking in a 65-nm LP CMOS technology. The novel topology has also been adopted in the design of a $15-\mathrm{GHz}$ divide-by-three for a frequency synthesizer targeting $5 \mathrm{G}$ specifications. In post-layout simulations at $100^{\circ} \mathrm{C}$, the circuit reaches a $23.6 \%$ locking range at $1.56-\mathrm{mW}$ DC power, featuring one of the best performance among divide-by-three ILFDs and, using just a single inductor, it occupies only $0.09 \mathrm{~mm}^{2}$.

\section{ACKNOWLEDGMENT}

The authors would like to thank Prof. Carlo Samori for insightful discussions and Keysight Technologies for the donation of Momentum software license.

\section{REFERENCES}

[1] H.-H. Hsieh et al., "A V-band divide-by-three differential direct injection-locked frequency divider in 65-nm CMOS," in Proc. Custom Integr. Circuits Conf. (CICC), Sep. 2010, pp. 1-4.

[2] J.-W. Wu, C.-C. Chen, H.-W. Kao, J.-K. Chen, and M.-C. Tu, "Divideby-three injection-locked frequency divider combined with divide-bytwo locking," IEEE Microw. Wireless Compon. Lett., vol. 23, no. 11, pp. 590-592, Nov. 2013.

[3] J. Kim, S. Lee, and D.-H. Choi, "Injection-locked frequency divider topology and design techniques for wide locking-range and high-order division," IEEE Access, vol. 5, pp. 4410-4417, 2017.

[4] S.-L. Jang, W.-C. Cheng, and C.-W. Hsue, "Wide-locking range divideby-3 injection-locked frequency divider using sixth-order $R L C$ resonator," IEEE Trans. Very Large Scale Integr. (VLSI) Syst., vol. 24, no. 7, pp. 2598-2602, Jul. 2016

[5] S.-L. Jang, Y.-S. Chen, C.-W. Chang, and C.-C. Liu, "A wide-locking range $\div 3$ injection-locked frequency divider using linear mixer," IEEE Microw. Wireless Compon. Lett., vol. 20, no. 7, pp. 390-392, Jul. 2010.

[6] A. Garghetti, A. L. Lacaita, and S. Levantino, "A low-power and wide-locking-range injection-locked frequency divider by three with dual-injection divide-by-two technique," in Proc. IEEE Int. Symp. Circuits Syst. (ISCAS), May 2018, pp. 1-4.

[7] Y.-L. Yeh and H.-Y. Chang, "Design and analysis of a $W$-band divideby-three injection-locked frequency divider using second harmonic enhancement technique," IEEE Trans. Microw. Theory Techn., vol. 60, no. 6, pp. 1617-1625, Jun. 2012.

[8] B. Razavi, "A study of injection locking and pulling in oscillators," IEEE J. Solid-State Circuits, vol. 39, no. 9, pp. 1415-1424, Sep. 2004.

[9] C. Y. Wu and C. Y. Yu, "Design and analysis of a millimeter-wave direct injection-locked frequency divider with large frequency locking range," IEEE Trans. Microw. Theory Techn., vol. 55, no. 8, pp. 1649-1658, Aug. 2007.
[10] S.-L. Jang, T.-C. Kung, and C.-W. Hsue, "Wide-locking range divide-by3 injection-locked frequency divider through enhanced 2nd harmonic," IEEE Microw. Wireless Compon. Lett., vol. 26, no. 7, pp. 537-539, Jul. 2016.

[11] Y.-H. Chang and Y.-C. Chiang, "A divide-by-3 injection-locked frequency divider in $0.18 \mu \mathrm{m}$ CMOS process for $\mathrm{K}$ band applications," in IEEE MTT-S Int. Microw. Symp. Dig., May 2015, pp. 1-3.

[12] W.-C. Lai, S.-L. Jang, and J.-W. Jhuang, "An injection-locked frequency divider by three with switching cross-couple architecture," in Proc. IEEE Int. Symp. Radio-Freq. Integr. Technol. (RFIT), Aug./Sep. 2017, pp. $159-161$.

[13] S.-L. Jang, W.-C. Lai, S.-S. Tzeng, and C.-W. Hsue, "A wide-band divide-by-3 injection-locked frequency divider using tunable MOS resistor," in Proc. IEEE Asian Solid-State Circuits Conf. (A-SSCC), Nov. 2015, pp. 1-5.

[14] B.-E. Seow, T.-H. Huang, C.-Y. Wu, P.-Y. Pao, and H.-R. Chuang, "A low-voltage 30-GHz CMOS divide-by-three ILFD with injection-switched cross-coupled pair technique," IEEE Trans. Microw. Theory Techn., vol. 65, no. 5, pp. 1560-1568, May 2017.

[15] K.-H. Chien, J. Y. Chen, and H.-K. Chiou, "Designs of K-band divideby-2 and divide-by-3 injection-locked frequency divider with Darlington topology," IEEE Trans. Microw. Theory Techn., vol. 63, no. 9, pp. 2877-2888, Sep. 2015.

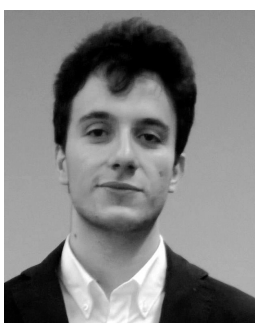

Alessandro Garghetti was born in Milan, Italy, in 1992. He received the M.S. degree in electronic engineering from the Politecnico di Milano, Milan, in 2017. In 2017, he joined SK Hynix Inc. as an Ana$\log$ Design Engineer, where he is actually involved in the design of high-density memory solutions for computing and mobile applications. His current interests are mainly focusing on analog ICs for wireless applications and 3-D NAND flash memories.

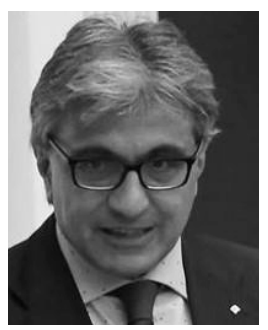

Andrea L. Lacaita (M'89-SM'94-F'09) received the Laurea degree (cum laude) in nuclear engineering from the Politecnico di Milano in 1985. From 1987 to 1992 , he was a Researcher with the CNR (Italian National Research Council). Since 1992, he has been a Professor with the Politecnico di Milano and a Full Professor since 2000. He was a Visiting Scientist at AT\&T Bell Laboratories, Murray Hill, NJ, USA, from 1989 to 1990, at the IBM T. J. Watson Research Center, Yorktown Heights, NY, USA, in 1999, and at the Data Storage Institute, Singapore, in 2011. He has co-authored over 350 papers in international journals and conferences. He has contributed to physics and technology of single photon avalanche diodes, reliability of nonvolatile memory devices, and design of low-noise fully integrated VCO's and frequency synthesizers for wireless applications. He has co-authored Integrated Frequency Synthesizers for Wireless Systems (Cambridge University Press, 2007). His current research interests include frequency synthesizers and wireless transceivers.

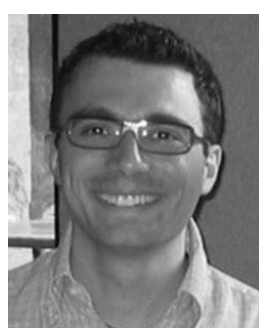

Salvatore Levantino (S'99-M'02-SM'16) received the Laurea degree (cum laude) and the Ph.D. degree in electrical engineering from the Politecnico di Milano, Milan, Italy, in 1998 and 2001, respectively.

He was a Consultant at Bell Labs, Lucent Technologies Inc., Murray Hill, NJ, USA, from 2000 to 2002. Since 2005, he has been an Assistant Professor and subsequently an Associate Professor of electrical engineering with the Politecnico di Milano. He has co-authored over 100 scientific papers and Integrated Frequency Synthesizers for Wireless Systems (Cambridge Univ. Press, 2007). He holds six patents.

Dr. Levantino is a Distinguished Lecturer of the IEEE. He is currently a member of the TPC for the IEEE Radio Frequency Integrated Circuits (RFIC) Symposium and the European Solid-State Circuits Conference, and a Committee Member of the Student Research Preview Program for the IEEE Solid-State Circuits Conference. He was a Steering Committee Member of the RFIC Symposium from 2015 to 2017. He was a Guest Editor for the May 2016 issue of the IEEE Journal of Solid-STATE Circuits and an Associate Editor for the IEEE TRANSACTIONS ON CIRCUITS AND SYSTEMS-I from 2014 to 2015 and for the IEEE TRANSACTIONS ON Circuits AND Systems II from 2012 to 2013. 